\title{
Antipode viticole ? Commerce global et terroirs d'invention en Nouvelle-Zélande
}

\section{Rory Hill}

\section{OpenEdition}

\section{Journals}

Édition électronique

URL : https://journals.openedition.org/geohist/2337

DOI : 10.4000/geohist.2337

ISSN : 2264-2617

Éditeur

Association française de la Revue de géographie historique

Référence électronique

Rory Hill, « Antipode viticole? Commerce global et terroirs d'invention en Nouvelle-Zélande », Revue de géographie historique [En ligne], 19-20 | 2021, mis en ligne le 20 novembre 2021, consulté le 21 novembre 2022. URL : http://journals.openedition.org/geohist/2337 ; DOI : https://doi.org/10.4000/ geohist.2337

Ce document a été généré automatiquement le 21 novembre 2022.

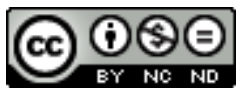

Creative Commons - Attribution - Pas d'Utilisation Commerciale - Pas de Modification 4.0 International - CC BY-NC-ND 4.0

https://creativecommons.org/licenses/by-nc-nd/4.0/ 


\section{Antipode viticole? Commerce global et terroirs d'invention en Nouvelle-Zélande}

Rory Hill

\section{Introduction}

1 Par rapport aux régions viticoles françaises, le vignoble néo-zélandais est récent, petit, et pauvre en traditions. Son industrie viti-vinicole regorge d'innovations, que ce soit dans les étapes techniques de la vinification (par exemple, l'osmose inverse), ou bien dans l'usage de capsules à vis, qui est presque universel. Aucune subvention n'est accordée aux viticulteurs par le gouvernement du pays (si ce ne sont celles d'exception liées à la pandémie Covid-19) : l'industrie est accrochée nettement au marché mondial et sujette à ses aléas. Pendant ces vingt dernières années, l'industrie viti-vinicole néozélandaise a eu le vent en poupe, avec un succès fou pour son vin blanc issu du cépage sauvignon blanc. Elaboré principalement dans la région de Marlborough, ce vin, commercialisé sous de nombreuses marques telles Cloudy Bay, Oyster Bay, Kim Crawford ou Matua, a trouvé ses amateurs aux Etats-Unis, au Royaume-Uni, et en Australie; et ces trois marchés demeurent de loin les plus importants pour le pays (New Zealand Winegrowers, 2020a : 36-37).

Or, ce marché mondial auquel le vignoble néo-zélandais doit son succès, et l'essor de la région de Marlborough par rapport aux autres régions viticoles du pays, ont déclenché des questions sur le destin de l'industrie. A l'échelle mondiale, dans un contexte où les containers de vin en vrac se déplacent partout, les supermarchés et les négociants sont devenus tout-puissants (Rainer et al, 2019), une course à la sous-enchère a suscité un cri de quo vadis? de la part de Michael Cooper, expert en vins néo-zélandais, dans un article récent (Cooper, 2021). Certes, le prix de revient pour un litre de sauvignon blanc de Marlborough en vrac, est coté par le négociant californien Ciatti (2021) à 3,50 euros, tandis qu'un litre de chardonnay d'Espagne ou d'Afrique du Sud l'est à 0,70 euro. Or ce 
chiffre n'assure ni un bel avenir pour l'industrie, au regard de ses coûts de production, ni une mise en valeur de maisons néo-zélandaises, d'autant que des gros lots de vin sont souvent vendus sous des marques déposées par des supermarchés étrangers.

3 Sous le déferlement des vins de Marlborough, les autres régions viticoles du pays (figure 1) ont le défi de se faire voir, se faire entendre, et se faire reconnaître par les consommateurs de vin domestiques et internationaux. Les innovations technologiques facilitent la grosse production du sauvignon blanc d'un certain style. Sauvignon blanc de Marlborough se prononce, par métonymie, vin de Nouvelle-Zélande. Mais son succès est tel que l'on est arrivé à une situation où $93 \%$ du vin néo-zélandais sont produits par 7\% des maisons (Cooper, 2021). On ne s'étonne pas des murmures dans l'industrie sur la désirabilité et la longévité de cette poule aux œufs d'or.

Figure 1 : Carte des principales régions viticoles du pays.

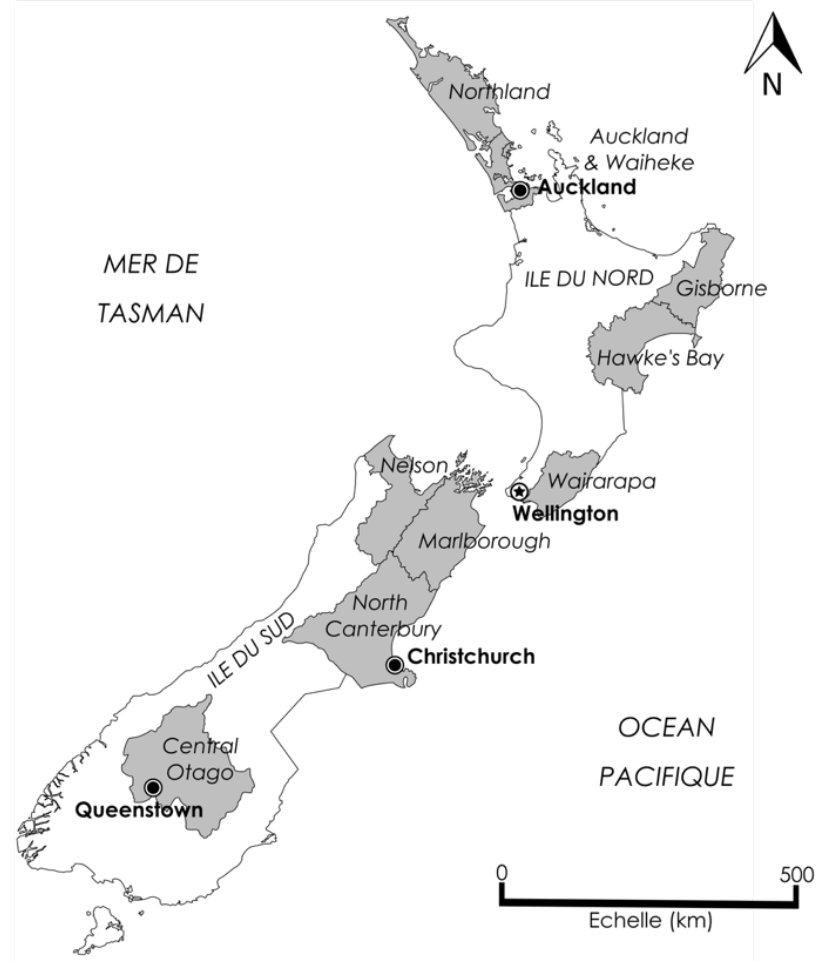

Réalisée par l'auteur sur les bases de la carte de AMitchell125 sur WikiMedia Commons

(URL : https://commons.wikimedia.org/wiki/

File:Geographical_indications_register_for_New_Zealand_wine.svg)

Le géographe Glenn Banks (2014) a noté que, par rapport aux vins australiens, les divers vins néo-zélandais font souvent référence à leur lieu d'origine. Issus d'un pays accidenté, touristique, et marqué par la culture Maori indigène, ces vins transmettent des histoires particulières par le biais de leurs labels. Or, ces efforts de reconnaissance de production locale, voire de terroir, doivent affronter une logique de production de masse, liée à l'histoire et aux mentalités agricoles du pays. Pour les maisons de vin de petite ou moyenne taille, on voit de nombreux efforts pour se différencier par la particularité de leur situation géographique, leurs choix de cépages, les sols et le microclimat dont elles disposent, l'expérimentation dans la vinification, la personnalité $\mathrm{du}$ vigneron, et leur connexion avec l'histoire culturelle et agricole de leur région. 
Prenons, par exemple, Mysterious Diggings, qui évoque l'héritage minier de la région de Central Otago, ou Craggy Range, évoquant les collines autour du pic Te Mata en Hawke's Bay, ou bien Johanneshof, un domaine qui marie des connaissances des vins aromatiques allemands aux terroirs accidentés des limites nord de Marlborough. La patrimonialisation évoquée par Boltanski et Esquerre (2017), Van Waerbeke (2001) ou Héritier (2012), et élaborée dans le contexte vinicole par Gade (2004), Crenn et Téchoueyres (2005), Demossier (2012) et autres, a aussi eu lieu en Nouvelle-Zélande. Elle a davantage d'importance pour ceux qui ambitionnent de produire de vins fins, voire de grands vins. L'histoire du vin Coleraine, par exemple, le vin rouge «le plus recherché » du pays (Renall, 2017 : 135), ne date que de 1982, mais son nom - un lieu en Irlande d'où venait la famille qui a fondé le domaine - évoque un pays qui stimule l'imagination et rappelle l'histoire de nombreuses familles immigrantes dont la plupart des Néo-zélandais sont issus. Bien que les histoires viticoles soient plus courtes que celles des régions viticoles du Vieux Monde, être pionnier est toujours possible, inventer des traditions est ouvert à tous, faire allusion ou rendre hommage aux cultures lointaines n'est pas rare. Qui plus est, la notion du temps a été interprétée de façon créative : en soulignant des moments forts de l'histoire récente, mais aussi par référence au temps géologique ou à l'usage de vieilles vignes (plus de 20 ans). Pour les producteurs à grande ou à moyenne échelle, innover peut bien dire interpréter le palimpseste du lieu pour trouver sa propre histoire, voire inventer de nouvelles traditions.

Dans cet article, nous caractériserons la trajectoire historique du secteur viti-vinicole en Nouvelle-Zélande, et nous interrogerons les rôles que jouent l'innovation et la tradition. Nous verrons comment les connaissances scientifiques et les avancées techniques ont aidé le développement du vignoble en de nombreuses régions du pays, et comment ce développement est aidé par le succès commercial mondial du vin de Marlborough dès les années 1980. Par l'étude d'une région émergente, nous analyserons la façon dont la culture et le commerce de vins s'entremêlent dans le pays, et nous démontrerons comment l'industrie viticole de la Nouvelle-Zélande comporte des similitudes avec celle du Vieux Monde.

\section{Les origines de la vigne au XIXè siècle}

6 Les tout premiers pieds de vigne furent plantés dans ce territoire reculé par le missionnaire Samuel Marsden, à Kerikeri, en 1819. Quelques années après, c'est le «Resident » ou gouverneur britannique James Busby qui s'occupait de la culture des vignes à Waitangi vers 1830. Amateur de vins, il s'occupait également de la vinification, et l'explorateur français Dumont-Durville a dégusté l'une de ses premières cuvées lors de son passage en 1840. Ces premiers essais se déroulèrent à l'extrémité nord de la Nouvelle-Zélande, où circulaient les marins, les marchands, les missionnaires, les pirates et les pêcheurs de baleine. Le climat y est très doux et le taux de pluviométrie élevé. Plus au sud, sur la presqu'île de Banks, ce furent des colons français qui plantèrent des vignes pendant les années 1840 dans le cadre de leur petite ville à Akaroa (figure 2). Effectivement, les Français ont joué un rôle important dans l'établissement du vignoble néo-zélandais. Des frères maristes ont établi la Mission Estate en 1851, et celle-ci demeure la plus ancienne maison de vins du pays. L'un de ses vins remporta un prix à l'exposition de Paris en 1892. Par ailleurs, un vigneron français 
cultivait des vignes et élaborait des vins dans la région reculée de Central Otago, où travaillaient des mineurs d'or pendant les années 1860 (Dalley, 2008).

Figure 2 : Tableau d'Akaroa vers 1860 par William Watkins.

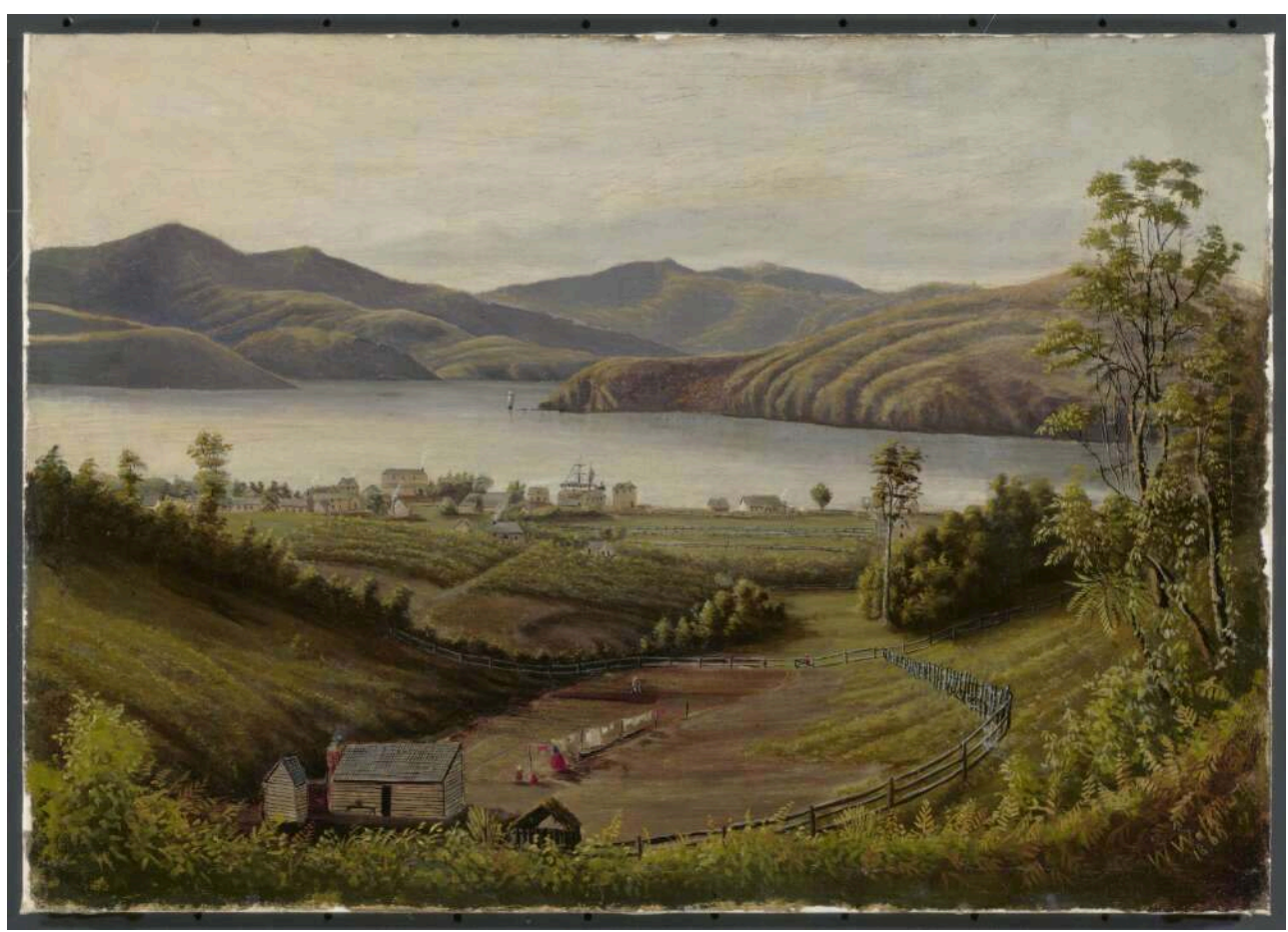

Le vignoble se présente derrière le village qui longe le lac.

Source : National Library of Australia.

7 Cette production de petite envergure attirait peu l'attention jusqu'au moment où, en 1895, le gouvernement de Nouvelle-Zélande, plus stable à la suite du traité de Waitangi, invita Romeo Bragato, scientifique et expert en viticulture, en vue d'analyser les possibilités d'en faire une production plus vaste. A la fin du XIX ${ }^{\mathrm{e}}$ siècle, la NouvelleZélande exportait de la viande, du beurre, de la laine, et du bois vers le Royaume-Uni. L'invention de la chaine du froid a en effet créé de grandes opportunités pour ce territoire fertile et éloigné de tout. Le gouvernement local fut soucieux de trouver d'autres commodités pour gagner de l'argent, et Bragato découvrit d'autres régions où l'on pouvait faire pousser de la vigne avec de bons rendements. Il repéra aussi le phylloxera près d'Auckland et donna des recommandations à cet égard. Pour diffuser ses idées, il publia son ouvrage Viticulture in New Zealand en 1905, et il retourna plusieurs fois en Nouvelle-Zélande dans les années qui suivirent.

Bien que les augures fussent bons durant les premières décennies du $\mathrm{XX}^{\mathrm{e}}$ siècle, il $\mathrm{y}$ avait plusieurs contraintes à l'expansion du vignoble néo-zélandais. L'expertise manquait, les traditions aussi, évidemment. Les terres étaient dédiées à l'élevage des moutons et des vaches, et des vins peu chers venus de l'Australie concurrençaient fortement la petite production vinicole locale. Qu'il y eût d'excellents terroirs à découvrir, c'est la culture de l'époque qui les a ignorés. Contrairement aux sociétés françaises et méditerranéennes, où le rôle culturel du vin assurait sa production presque partout (Dion, 1959), le vin ne fut pas la boisson du peuple en NouvelleZélande. Peu de membres de la société coloniale appartenaient aux classes aisées, 
auxquelles les vins furent généralement destinés, selon la culture britannique de l'époque. Si l'on compare la consommation annuelle de vin par personne en France et au Royaume-Uni pendant les années 1920, par exemple, les chiffres donnent 151,4 litres par personne en France contre seulement 1,5 litre par personne outre-Manche (Anderson et al, 2017 : 192-93). Dans les sociétés coloniales d'Irlande, de Canada et de Nouvelle-Zélande, les données sont encore plus faibles : 1 litre, 0.7 litre, et 1,1 litre, respectivement. Dans un tel contexte, ce n'est pas le vin qui fait bon commerce : la bière est plus appréciée. Il y a aussi certaines tendances protestantes qui refusent l'alcool sous n'importe quelle forme. Entre les années 1911 et 1987, pas moins de 24 référendums ont eu lieu en Nouvelle-Zélande sur la question de la vente d'alcool. Sous l'influence des mouvements de "Temperance », y compris les Méthodistes, et avec un œil sur la Prohibition aux Etats-Unis, une part de la société néo-zélandaise fut mal à l'aise avec le vin, la bière et les spiritueux pendant longtemps.

\section{Le début d'une véritable industrie}

S'ensuivent de grands changements à la fois dans la culture et dans la production viticole du pays. Qu'est-ce qui les a déclenchés? L'histoire qui est souvent racontée (voir Schuster et al (2002: 31-32), est la suivante: à la fin de la Seconde Guerre Mondiale, à laquelle de nombreux militaires des colonies britanniques ont participé, les soldats retournèrent chez eux avec de nouvelles habitudes et de nouveaux goûts qu'ils avaient découverts en Europe. Parmi eux, un goût pour le vin. Ce n'était plus le goût des Anglais pour des vins doux et fortifiés, tels les vins de Porto ou de Madère (et les vins comparables issus des autres lieux), mais un goût pour les vins de table, qui seront pris lors d'un repas. Entre 1940 et 1960, des lois permettant aux restaurants de vendre de l'alcool sont promulguées. Par ailleurs, les connaissances sur le vin se diffusent en même temps que les revenus disponibles s'accroissent. Ce sont les signes d'une culture qui change, laquelle place l'alcool dans un contexte de consommation modérée, voire " civilisée ", comme l'observait D W Beavan en 1977 (p. 4), préférable aux coutumes de consommation « barbares » visibles dans les tavernes.

Les entrepreneurs de l'industrie viticole se développent près d'Auckland, la ville la plus importante du pays. Les cépages varient (les choix des viticulteurs étant libres), la technologie s'impose, et les techniques de l'industrie australienne exercent leur influence. Chez Corban, une maison fondée par un Libanais au début du siècle, on commence à utiliser les cuves en inox en 1958, essentiellement dans une optique de production hygiénique et efficace. Cinq années plus tard, Corban assure les premières exportations de vin à large échelle. Pendant les années 1960 et 1970, la production générale s'accroît, et s'adapte aux goûts qui changent. Au début des années 1960, seulement $12 \%$ de la production néo-zélandaise sont des vins non fortifiés; au début des années 1980, le chiffre atteint 73\% (Dalley, 2008). Effectivement, dans les années 1970, on constate le début d'un essor de consommation de vin dans le monde anglophone qui perdure jusqu'aux années 2010 (Legouy et Boulanger, 2015 ; Hill, 2016). L'expert D W Beaven (p. 53) a remarqué la tendance en 1977 :

11 «Dès les années 1960, l'industrie viticole néo-zélandaise s'est concentrée peu à peu sur l'utilisation de cépages de Vitis vinifera, et les attitudes des consommateurs ont changé à un tel point qu'il y a maintenant un marché considérable pour les vins. L'expérience de voyages internationaux, l'arrivée des touristes, et davantage d'échanges avec le marché 
australien (qui est plus développé et plus sophistiqué), ont fait que les Néo-zélandais demandent des vins de meilleure qualité, issus des raisins de meilleure qualité. » ${ }^{1}$

Pendant les années 1970, on élabore des vins des cépages tels que le Müller-Thurgau et le Palomino, et on commence à cerner les aptitudes viticoles des diverses régions du pays (Moran, 2016 : 46-53). De nouvelles expériences dans la région de Marlborough furent faites, ainsi que dans les régions de Canterbury, Wairarapa, et Hawke's Bay (figure 1). Des agronomes, des scientifiques et des amateurs de vin participent à ces missions : ils observent les conditions climatiques, pédologiques et topographiques, et les mettent en relation avec leurs connaissances d'autres vignobles. De plus en plus de données émanent de l'Australie et de la Californie, et les nouveaux vignobles sont pour la plupart irrigués à l'australienne, nonobstant les conditions moins chaudes en Nouvelle-Zélande. Peu à peu, les viticulteurs abandonnent la région d'Auckland, et s'installent dans de nouvelles régions, où les conditions climatiques sont plus idéales (plus sèches, au moins), et où les terres se vendent moins cher. Dans l'Ile du Nord, ce sont les régions de Hawke's Bay et Gisborne qui voient les vignobles s'agrandir; dans l'Ile du Sud, c'est Marlborough, avec un peu d'activité dans les régions de Nelson et Canterbury. On fait des essais avec de nombreux cépages, y compris des hybrides, et il est remarqué que les Sauvignon blanc, issus des terroirs de Marlborough, portent des saveurs de fruits brillants et exotiques, accompagnés d'une belle fraîcheur.

Dès les années 1980, ces vins de Marlborough font sensation dans le monde anglophone. Sous les marques de Matua, Kim Crawford, Cloudy Bay et autres, ces vins blancs aux saveurs intenses se vendent comme des petits pains sur les marchés australiens, américains et britanniques, et remportent le grand prix du Sunday Times Wine Festival entre 1986 et 1988 (Lewis et Le Heron, 2019 : 121). L'économie néo-zélandaise se tourne définitivement vers le monde entier à cette époque, d'autant plus que le Royaume-Uni a abandonné ses anciens privilèges de commerce en 1973 pour entrer dans le Marché Commun européen. Le Japon et la Chine s'intéressent à leurs ressources primaires, et Hollywood découvre ses paysages sauvages pour des films d'aventure tels Willow. Dans un esprit néo-libéral, le gouvernement néo-zélandais n'accorde plus de subventions ni à ses agriculteurs ni à ses viticulteurs. Or, en vendant de plus en plus à l'export, et forts des beaux millésimes des années 1980, les entrepreneurs du vin multiplient les succès. La superficie du vignoble augmente, jusqu'au point que l'on mène une campagne d'arrachage de 25\% du vignoble en 1986 (Dalley, 2008; Moran, 2016: 40-46). Les viticulteurs et les vignerons s'empressent de maîtriser les autres cépages, et le pinot noir se voit planté dans la région de Central Otago, jusqu'alors un territoire occupé par les moutons et les vergers.

14 A la fin du siècle, les Néo-zélandais boivent en moyenne 15 litres de vin par personne (Anderson et al, 2017 : 192), et les vins du pays se vendent partout dans le monde, remportant des médailles et de belles appréciations de la part des experts. Sur le marché mondial, les vins néo-zélandais paraissent excellents sur le plan technique, appétissants et abordables sur le plan organoleptique (à boire jeunes, en général), et stimulants sur le plan de l'imagination. Leur concurrence avec les vins français est souvent indirecte - il y a peu de vins rouges, et les réputations de grands vins manquent - mais ils font partie d'une vague de vins du Nouveau Monde qui rivalisent avec ceux du «Vieux Monde » à cette époque. Le vin se démocratise de plus en plus, et les consommateurs à Londres, à Los Angeles ou à Sydney commandent des verres par référence à leur cépage et non plus par référence à leur région d'origine. Ce 
phénomène rend la réputation des anciennes régions viticoles moins importante, et ouvre le marché à n'importe quel pays qui peut fournir des vins de tel ou tel cépage. Il remet en cause la notion de terroir - fondée sur un lieu précis et une mode de culture traditionnelle - et il fut le sujet d'un débat entres les géographes autour de l'année 2000 (Bailly, 2000 ; Pitte, 2001 ; voir aussi Schirmer, 2007).

Le vin fait partie de l'art de vivre d'un plus grand nombre de consommateurs néozélandais, et l'œnotourisme se développe. Le premier restaurant attaché à un domaine viticole ouvre ses portes en 1979, et la curiosité générale pour le vin s'accroît. Ailleurs dans l'océan Pacifique, les premières tentatives de production de vin se développent sur les îles touristiques de Tahiti et de Hawaï, et celles-ci nous rappellent jusqu'à quel point le vin est apprécié dans le contexte informel et expérientiel des vacances (la consommation de vin étant définitivement entrée dans la "experience economy » de Pine et Gilmore (1998)). Sur le plan technique, les viticulteurs néo-zélandais remplacent leurs cépages hybrides (tel le Müller-Thurgau) par des cépages nobles (tel le Chardonnay), et les "flying winemakers » français et autres, sont de plus en plus invités aux domaines. La thèse sur la mondialisation du vin évoquée par Jonathan Nossiter dans son film Mondovino (2004) n'épargne pas la Nouvelle-Zélande, ni dans son commerce mondial, ni dans son ouverture aux techniques d'ailleurs. Rappelons qu'aucun cépage n'est indigène à la Nouvelle-Zélande, et que le pays a exporté davantage de denrées qu'il n'en consommait pendant longtemps: cerises, kiwis, pommes, avocats, moutons, venaison vers les pays demandeurs (Chine, Japon, Amérique ou Europe). En 1999, les supermarchés ont finalement été autorisés à vendre du vin, ce qui marque une étape importante dans le développement de la culture de consommation de vin dans le pays; étape que l'on pourrait qualifier de «normalisation».

\section{Les folles années : un secteur viti-vinicole qui se divise}

Dans les premières années du XXI siècle, trois phénomènes surviennent. D'abord, le gouvernement de Nouvelle-Zélande lance son «100\% Pure New Zealand " campagne pour le secteur du tourisme; ensuite, les films de la série Le Seigneur des Anneaux, tournés en Nouvelle-Zélande, se diffusent dans le monde entier; enfin, le prix de revient du vin blanc de Marlborough atteint un niveau qu'il n'atteindra plus jamais. Ces trois phénomènes sont bien liés : le rayonnement mondial de la Nouvelle-Zélande est en hausse, et son vin porte des images positives. La production du Sauvignon blanc de Marlborough trouve sa formule: irrigation, gros rendements, vendanges mécanisées, aucun passage en fûts de chêne, mise en bouteille sous capsule à vis, mise en vente rapide. Des journalistes parlent d'un «Sauvalanche" (avalanche de Sauvignon blanc) coulant de Marlborough (par exemple, Hawke's Bay Today, 2008), et des fortunes se réalisent dans l'industrie, avec des acquisitions et des cotations en bourse. La société Montana Wines, acquiert Corban en 2000, devenant ainsi la plus grosse société viticole du pays. Un an après, Montana est acquise par le holding anglais Allied Domecq, et en 2005, ce dernier est acquis à son tour par Pernod Ricard. La « fin de l'histoire » qu'a proposée Francis Fukuyama aurait pu, peut-être, s'appliquer à ce stade du développement de l'industrie viticole en Nouvelle-Zélande. Or, ailleurs dans le pays, 
l'histoire est différente, et derrière le succès fulgurant de Marlborough, des problèmes s'accumulent, lesquels ont été récemment évoqués par Michael Cooper (2021).

La superficie du vignoble national est passée de 6000 hectares en 1990 à 40000 hectares en 2020 (Moran, 2000 ; New Zealand Winegrowers, 2021). La plupart de ces nouvelles parcelles se trouvent à Marlborough, et sont plantées en sauvignon blanc. L'industrie est alors de plus en plus déséquilibrée. Le cabernet sauvignon et le merlot sont cultivés dans les régions d'Auckland (notamment à l'Ile Waiheke) et Hawke's Bay. La syrah pousse aussi à Hawke's Bay. Le pinot noir, quant à lui, trouve ses terroirs d'excellence dans le Wairarapa, North Canterbury, et Central Otago. Le problème est que, sur le plan mondial, il y a trop de concurrents dans ce genres de vin -y compris de gros producteurs en Australie. En Nouvelle-Zélande, où le domaine moyen ne mesure que 20 hectares (New Zealand Winegrowers, 2021) - chiffre encore plus faible dans les régions qui se concentrent sur les vins rouges - les économies d'échelle ne sont pas de mise. Dans un rapport récent de la part des comptables Deloitte (2019: 15), les petits producteurs de vins néo-zélandais (aux revenus annuels inférieurs à 900000 euros) peinent à gagner un profit de $7 \%$ avant impôts, tandis que les gros producteurs (aux revenus supérieurs à 12 millions d'euros) touchent $18,7 \%$ de profit. Pendant que des grandes entreprises tel Delegat se cotent en bourse, des petites ferment leurs portes.

Pour beaucoup de vignerons néo-zélandais, le chemin vers la qualité passe par la perfection technique, et par la reconnaissance des autres. Dès le début du XXI siècle, Villa Maria, l'une des plus grandes maisons, abandonne les bouchons et opte pour les capsules à vis. La plupart des grandes maisons l'ont suivie dans cette décision : le risque du désappointement occasionné par un vin bouchonné ne vaut pas la valeur traditionnelle de liège. Les innovations en vinification telles l'osmose inverse, la filtration tangentielle et la thermovinification trouvent leur place dans les grandes entreprises. Etant donné que ni les procédés de viticulture ni les étapes de vinification ne sont réglés par des cahiers de charges des appellations d'origine (ce qui est le cas dans certaines appellations françaises), d'éventuelles traditions régionales ne posent que peu de barrières à l'efficacité de la production et à l'adoption de nouvelles technologies. Pour le sauvignon blanc, du point de vue chimique, on a identifié les thiols qui donnent les saveurs typiques, et on adapte la viticulture et la vinification pour les maintenir dans les proportions que l'on souhaite. Pour la syrah, on évite à tout prix la présence des levures du genre Brettanomyces, puisque celle-là est considérée comme une faute par certains experts (bien que pour des autres, elle fasse partie de la typicité des grands syrahs tel le Châteauneuf-du-Pape). Prendre un risque pour un style idiosyncratique tenterait peu de grandes maisons, avec leurs contrats d'export, et avec l'idée que se font les consommateurs du caractère d'un vin de cépage (Schirmer, 2007). Faute de réputation de longue date, les médailles sont très recherchées et par les vignerons, et par les consommateurs. Hormis les sauvignons blancs de Marlborough, devenus l'archétype du vin de Nouvelle-Zélande, les divers vins du pays doivent tout faire pour se faire remarquer dans les rayons des supermarchés et des cavistes.

Pour les petits producteurs, il existe pourtant plusieurs stratégies pour réussir sur le plan commercial. Il y a la diversification d'activités. Le tourisme étant un secteur économique crucial pour le pays avant la Covid, l'œnotourisme s'est ainsi largement développé depuis la fin des années 1970. Chez Rippon, par exemple, ils tirent profit de leur superbe situation au bord du Lac Wanaka (Central Otago) pour accueillir non seulement des touristes, mais des convives lors des mariages et des anniversaires. Une 
grande partie de leur stock de vins se vend lors de tels évènements. D'autres domaines se tournent largement vers la valorisation de leur terroir : ils mettent en avant les particularités de leurs situations géographiques, de leurs sols, de leurs microclimats ; ils réclament des appellations d'origine (des GI); et ils défendent les traditions - aussi récentes qu'elles soient - de leurs domaines. En Hawke's Bay, on fait la promotion des Gimblett Gravels, ancien lit de rivière dans lequel sont élaborés les vins rouges fins de la région (Murray et Overton, 2011). En Central Otago, on met en valeur la typicité des pinots noirs des différents villages tels Bannockburn et Bendigo (New Zealand Winegrowers, 2020b : 30-32). Et en North Canterbury, les producteurs se flattent d'avoir des sols calcaires qui ressemblent à ceux de la Bourgogne (voir cas d'étude ci-dessous). Certains vignerons sont devenus célèbres : Tony Bish avec ses chardonnays de Hawke's Bay; James Milton avec ses vins biodynamiques de Gisborne ; Jenny Dobson, riche de ses expériences à Bordeaux. Certains vignerons ont parcouru le monde à la recherche de tel ou tel sol ou climat, et ils les ont trouvés en Nouvelle-Zélande (voir Moran, 2016 : 345-9). Dans un pays où l'avènement de la viticulture commerciale est récent, l'excellence peut encore se décliner sous plusieurs formes.

\section{Etude de cas : North Canterbury}

Certaines de ces tendances sont bien visibles dans le cas du vignoble de la région de North Canterbury (Cantorbéry du Nord). Région sèche aux alentours de Christchurch, dans l'Ile du Sud, l'exploitation commerciale de la vigne n'y débute qu'à la fin des années 1970, avec l'appui des expériences scientifiques faites à l'université de Lincoln (Schuster et al, 2002). Au fur et à mesure que les connaissances viticoles s'améliorent et que les consommateurs réclament des vins de qualité, le vignoble s'est agrandi, et il s'est concentré sur certains terroirs et certains cépages. La vigne y est cultivée dans trois sous-régions : la plaine, près de Christchurch ; la vallée de Waipara, dans le nord ; et la presqu'île de Banks. Toutes les trois ont des microclimats et des paysages différents. Dans la vallée de Waipara (figure 3), les terres sont protégées du vent frais de l'est par les collines de Teviotdale, mais elles restent ouvertes à l'effet de foehn du nord-ouest, ce qui rend le climat plus apte à la culture de la vigne par rapport à la plaine. Sur la presqu'île de Banks, on ne craint guère le gel, grâce au climat modéré engendré par l'océan qui l'entoure.

De 2000 à 2016, la superficie du vignoble à Waipara est passée de 210 à 1251 hectares, tandis qu'elle a diminué de 232 à 168 hectares sur la plaine (Overton, 2020). Il y eut de nombreux entrepreneurs qui lancèrent leur entreprise à Waipara pendant les années 2000 , remplaçant des pâtures par des vignobles, et certains vignerons de la plaine se sont même établis à Waipara. Son climat plus chaud et plus ensoleillé permettait de belles vendanges pour de nombreux cépages, sa géologie, avec des zones de calcaire, et sa topographie en pente (collines et côtes) rappelaient ceux de la Côte d'or de Bourgogne. Visible depuis la route nationale qui relie Christchurch et Marlborough, ce vignoble (figure 3) est la preuve d'une économie rurale qui change au cours de son histoire, comme l'attestait Warren Moran (2016:334), géographe et chroniqueur du vignoble néo-zélandais : pauvres, les précipitations moins fréquentes. $»^{2}$ 
Figure 3 : Vignoble à Waipara (Nouvelle-Zélande) durant l'été austral (janvier).

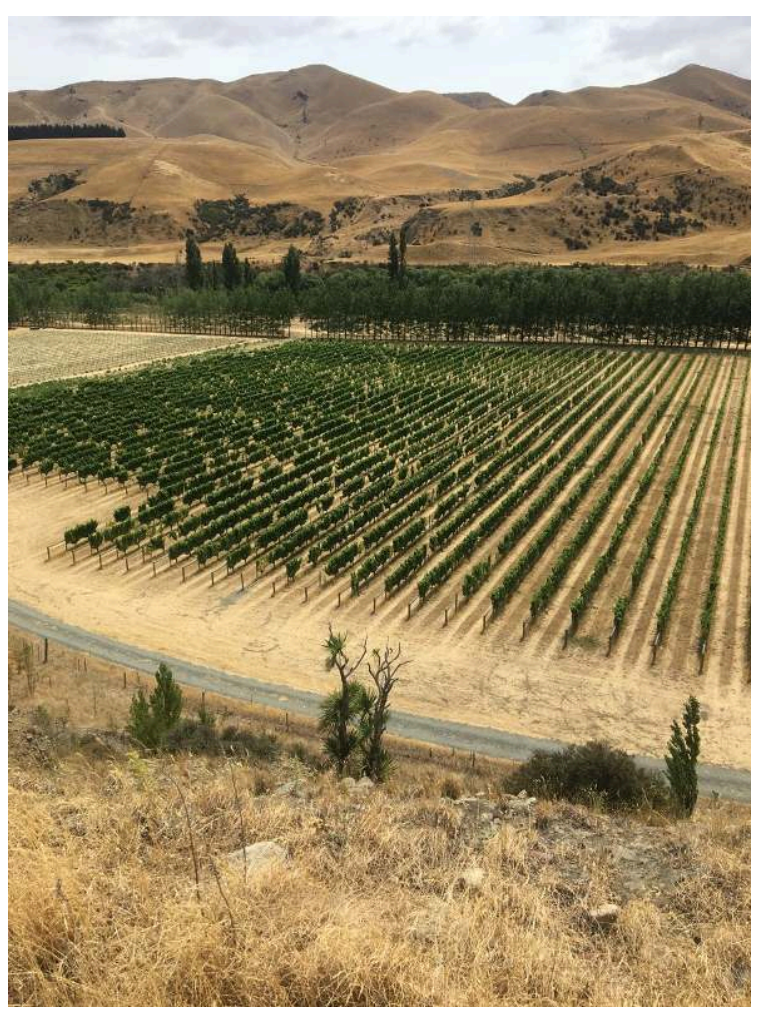

Parcelle de vignes de chardonnay, sauvignon blanc et riesling sur une ancienne plaine inondable. Vignes de 30 ans et certaines autres qui viennent d'être plantées. L'irrigation est nécessaire au regard des lits de gravier dans le sol (drainage).

Cliché : R. Hill, janvier 2020.

Le défi commercial que doivent affronter les vignerons de North Canterbury est considérable. Leur production ne représente que $4 \%$ du total national. Rappelons aussi que, sur la même île, se trouvent le vignoble de Marlborough avec son célèbre sauvignon blanc (75\% de l'encépagement), ainsi que le vignoble de Central Otago, de plus en plus réputé pour son pinot noir ( $75 \%$ de l'encépagement). Dans ces deux régions, l'encépagement est peu varié. En revanche, dans le North Canterbury, pinot noir et sauvignon blanc côtoient pinot gris, riesling et chardonnay. Bien que le pinot noir soit actuellement dominant, celui-ci ne recouvre que $31 \%$ du vignoble de la région (New Zealand Winegrowers, 2020c). Une telle diversité rend l'élaboration d'une réputation régionale plus difficile; d'autant plus difficile que la valorisation des vins des régions au nord et au sud est très axée sur leurs cépages «star ». Au cours des années 2000, un pourcentage du sauvignon blanc de North Canterbury fut destiné aux assemblages des maisons de Marlborough : le prix de revient des vins de Marlborough était en hausse ; les lettres de noblesse de North Canterbury manquaient.

De taille modeste, la plupart des domaines de North Canterbury cherchent à mettre en valeur leur terroir, puisqu'ils ne peuvent obtenir de grosses productions, comme le pratiquent d'ailleurs de nombreuses régions du Vieux Monde. Le vin Jaune et le Savagnin jurassiens en sont de bons exemples (Fassier-Boulanger, 2014). Ces domaines néo-zélandais louent les mérites de leurs parcelles et les styles de leurs vins, ils montent des festivals pour piquer la curiosité des habitants de Christchurch et d'ailleurs. Evoquer le terroir est pourtant compliqué. Dans un premier temps, les trois 
sous-régions (la plaine, Waipara, et la presqu'île de Banks) sont différentes des points de vue du paysage, du sol, et du climat. Evoquer le terroir de North Canterbury tout entier est difficile; or ce fut une étape nécessaire pour l'octroi de la Geographical Indication $^{3}$ (GI) - équivalent d'Indication Géographique Protégée (IGP), ou d'Appellation d'Origine Protégée (AOP). Dans le dossier pris en considération par l'instance du gouvernement de Nouvelle-Zélande (IPONZ), on voit des efforts pour borner les limites de la région en utilisant les rivières comme frontières naturelles, en faisant référence aux paramètres climatiques ou aux cartes dressées par des autres organisations (IPONZ, 2021a). L'instance interroge l'organisme de défense et de gestion - North Canterbury Winegrowers - sur sa logique, et le dossier est remis à jour. On n'y conteste pas la diversité géographique de la région, mais on insiste sur la validité de l'appellation North Canterbury avec des échos à la phrase «local, loyal et constant». A savoir : le sud de Canterbury n'a aucun vignoble; cette appellation viticole a été utilisée pendant quelques années d'une manière informelle; et une fragile notion de typicité - surtout pour les pinots noirs et les rieslings - commence à se faire entendre dans les cercles des amateurs de vin. Prenons, par exemple, cette critique d'un pinot noir d'un terroir de la presqu'île de Banks (Whistling Buoy, 2021) :

«Ce vin me fait frémir. C'est le vrai sens du terroir ; ce vin reflète son lieu d'origine, et je ressens les collines, le touradon, l'herbe, l'arôme extraordinaire du vent du nordouest sous un beau jour d'été. Ce vin est superbe $! »^{4}$

On a l'impression d'assister à l'épanouissement d'une tradition viticole dans cette région, même s'il a fallu des innovations pour y arriver : le système d'AOC (GI) en Nouvelle-Zélande n'entre en vigueur qu'en 2017 (IPONZ, 2021b), et une certaine part des moûts de la région furent utilisés dans l'assemblage des vins de Marlborough. Cette appellation de North Canterbury est finalement décernée en mars 2020. Victoire mitigée, au regard de la pandémie qui est arrivée en Nouvelle-Zélande quelques semaines après.

Il y a longtemps que la notion de terroir a franchi les limites de la France. Elle est reconnue par des amateurs du vin et des vignerons partout dans le monde (Trubek, 2008 ; Cappeliez, 2017 ; Hill, 2020). Or, évoquer le terroir, terme utilisé en français par ailleurs, ne convainc pas tous les vignerons. Dans le cours des interviews tenues avec une vingtaine de vignerons en North Canterbury en 2019 et 2020, on a appris que même si tous les vignerons reconnaissent le mot terroir, ils hésitent à l'utiliser dans la commercialisation de leurs produits. L'un d'entre eux confiait qu'il utiliserait le mot lors d'un dîner où les vignerons sont les invités, mais il ne l'utiliserait pas auprès des visiteurs réguliers de son domaine. Un autre démontrait l'informalité qui régnait dans la culture néo-zélandaise en racontant :

28 " [Le terroir] est bien pertinent, et les Français l'utilisent correctement ... il décrit très bien les choses. Or, nous ne l'avons pas retenu dans nos communications. En général, le langage que l'on utilise est plutôt informel... nous ne voulons pas être prétentieux (high falutin') dans notre style de communication. $»^{5}$

Dans un pays où le français n'est pas compris d'une large partie de la population, et dans le contexte d'un produit qui comporte une certaine image de luxe, un mot comme «terroir» peut intimider au lieu de donner envie. Or, la logique du terroir - la primauté du lieu - est adoptée par certains domaines tendant vers le haut de la gamme, soit pour plaire aux amateurs de vin, soit pour éduquer, en tout cas pour transmettre une passion. Black Estate et Mountford Estate sont deux domaines qui vinifient leurs 
parcelles séparément et vendent leurs vins sous le nom de la parcelle d'origine. Pour Black Estate, ils insistent sur les qualités des différentes parcelles lors des dégustations au domaine, et pour ceux qui ont commandé leur vin en ligne, la bouteille arrive enveloppée dans une carte topographique qui met en valeur les trois parcelles. Cette mise en avant de parcelles rappelle la Bourgogne, où les climats, connus dans certain cas dès le Moyen Âge, sont fort réputés et protégés. En Nouvelle Zélande, cependant, cette commercialisation de petits terroirs est une innovation contre l'ordre des choses et non pas une tradition de longue date.

Plus largement, l'utilisation du terroir, telle qu'on la pratique en North Canterbury, diffère de son usage en France. Le terroir n'évoque ni l'histoire ni la mythologie du vin, comme il peut le faire en France (Roudié, 2007 ; Laferté, 2011). Souvent, le terme prend un sens plus technique. Sur certains sites web des domaines de North Canterbury, le terroir est présenté en rapport avec le choix des clones de pieds de vigne. C'est l'éloge des connaissances agronomiques du viticulteur actuel qui est fait. Admettons que le langage des clones est moins poétique que celui des traditions, des grands vins, et de l'identité régionale contrairement au langage visant certains terroirs et stimulant l'imagination en France. Toutefois, il suggère un rapport entre les terres et les hommes qui reflète d'ailleurs ce qui se passait jadis dans les régions viticoles du Vieux Monde. Prenons, par exemple, les notes de dégustation d'une cuvée du domaine Tongue In Groove (2020) de North Canterbury:

«Ceci est notre troisième millésime de vin de la parcelle Cabal Vineyard, qui se trouve sur les pentes douces du nord de la vallée de Waipara. Le sol est argilo-calcaire. Divers clones y ont été plantés [...] et vinifier les clones séparément permet au vigneron de développer ses connaissances sur chaque clone. Les vignobles en Bourgogne sont généralement plantés avec un mélange de clones, tous vendangés en même temps. Or, les Bourguignons ont développé leurs connaissances sur les clones et les parcelles pendant des siècles. En Nouvelle-Zélande, nous n'avons que vingt ans d'expérience, et il nous reste beaucoup à apprendre. $»^{6}$

Une dernière remarque relative au terroir peut être mentionnée. En Nouvelle-Zélande, on assiste à la mobilisation des autres sens du terme «lieu ». Le mot tūrangawaewae, issu de la langue Maori, vient d'être adopté par certains dans l'industrie pour exprimer une connexion profonde à la terre (Vinography, 2017, par exemple). Parmi les vignerons de North Canterbury, certains considèrent que le tūrangawaewae est similaire au concept de terroir, même si tout le monde n'est pas d'accord. Le sens du mot est plus large dans la culture Maori, et l'utiliser dans le contexte commercial du vin n'est pas forcément bien vu. Il faut en outre se rappeler que, en ce qui concerne la connexion à la terre, les communautés Maori ont perdu la plupart de leurs terres ancestrales au cours des quelque deux cents ans de colonisation (Wynyard, 2019). Actuellement, les Maoris sont sous-représentés dans l'industrie viticole, bien que la toponymie, l'iconographie et même la mythologie de leur culture figurent souvent sur les labels des vins. Il y a pourtant de bons signes pour l'avenir. En North Canterbury, la maison TIKI Wines est sous la direction d'un vigneron Maori, et il fait partie d'un collectif national de vignerons Maori - Tuku - qui vient de se lancer. Dans le cadre d'une société qui se voit biculturelle, l'inclusion des Maoris dans le succès continu de l'industrie viticole est de première importance. Il sera très intéressant de voir comment les valeurs, les traditions et les innovations des deux cultures marqueront le vignoble dans les années qui viennent. 


\section{Conclusion} produits authentiques, porteurs d'histoires de terroir, forts de leurs réputations de longue date. De l'autre, le vin est devenu un plaisir quotidien, décliné sous plusieurs cépages faciles à comprendre et à apprécier. Le commerce du vin est global - que ce soit dans les circuits de ventes aux enchères pour les vins fins, ou dans les containers de vin standard, venant de n'importe où, destiné aux puissants supermarchés de l'Europe. Pendant que la consommation de vin en France a diminué ces quarante dernières années, elle a augmenté dans le monde anglophone, et elle touche toutes les classes sociales. Rappelons aussi que la qualité de vin s'est améliorée en général grâce aux avancées techniques et hygiéniques qui ont été largement adoptées dans tous les pays qui le produisent. technologiques, d'autant plus qu'il y a peu de traditions pour ralentir leur adoption. Comme l'a dit Moran (2000:549) :

«Tandis que dans l'industrie française, la qualité et la réputation des vins démontrent avec certitude que le travail des vignerons est efficace, les cultivateurs néo-zélandais ont bénéficié de la recherche théorique et empirique qui a amélioré leur compréhension de leurs nouveaux environnements. »

Dans les autres secteurs agricoles du pays, l'exemple des grands succès qu'ont rendu possible les avancées techniques a aussi formé les mentalités. A la fin du XIX siècle, l'implémentation de la chaîne du froid a permis l'exportation de la viande et l'expansion de l'élevage - activité prestigieuse, voire héroïque - presque partout dans le pays. Au milieu du XXe siècle, l'atomisation industrielle permet la préparation du lait en poudre, qui demeure lucratif jusqu'à présent. On ne s'étonnera donc pas que l'utilisation d'une technologie comme l'osmose inverse pour améliorer un vin puisse paraître intéressante pour certains vignerons. Et ce d'autant dans les grands domaines, et quand on cible un style de vin issu d'un certain cépage, et non pas une expression indélébile du terroir.

Or pour les petits, qui profitent à peine du «Sauvalanche » coulant vers les grands marchés d'export, on a vu qu'une élaboration de vin plus artisanale est possible, et que le terroir - qu'il soit compris d'une façon poétique ou technique - compte. Des traditions se forment, et la notion du temps lui-même a été élargie pour mettre en valeur l'âge des pieds de vigne ou les évidences des ères géologiques. On s'accroche également aux pionniers. Chez Te Mata, ils font référence à l'an 1891, quand furent plantés les premiers vignobles. La Mission Estate est fière de ses origines de l'an 1851. Et à Akaroa, la scène tranquille d'une ville avec son vignoble en arrière-plan qui se présentait aux yeux des Victoriens (figure 2), demeure (figure 4). 
Figure 4: Vignoble à Akaroa durant l'hiver austral (juin).

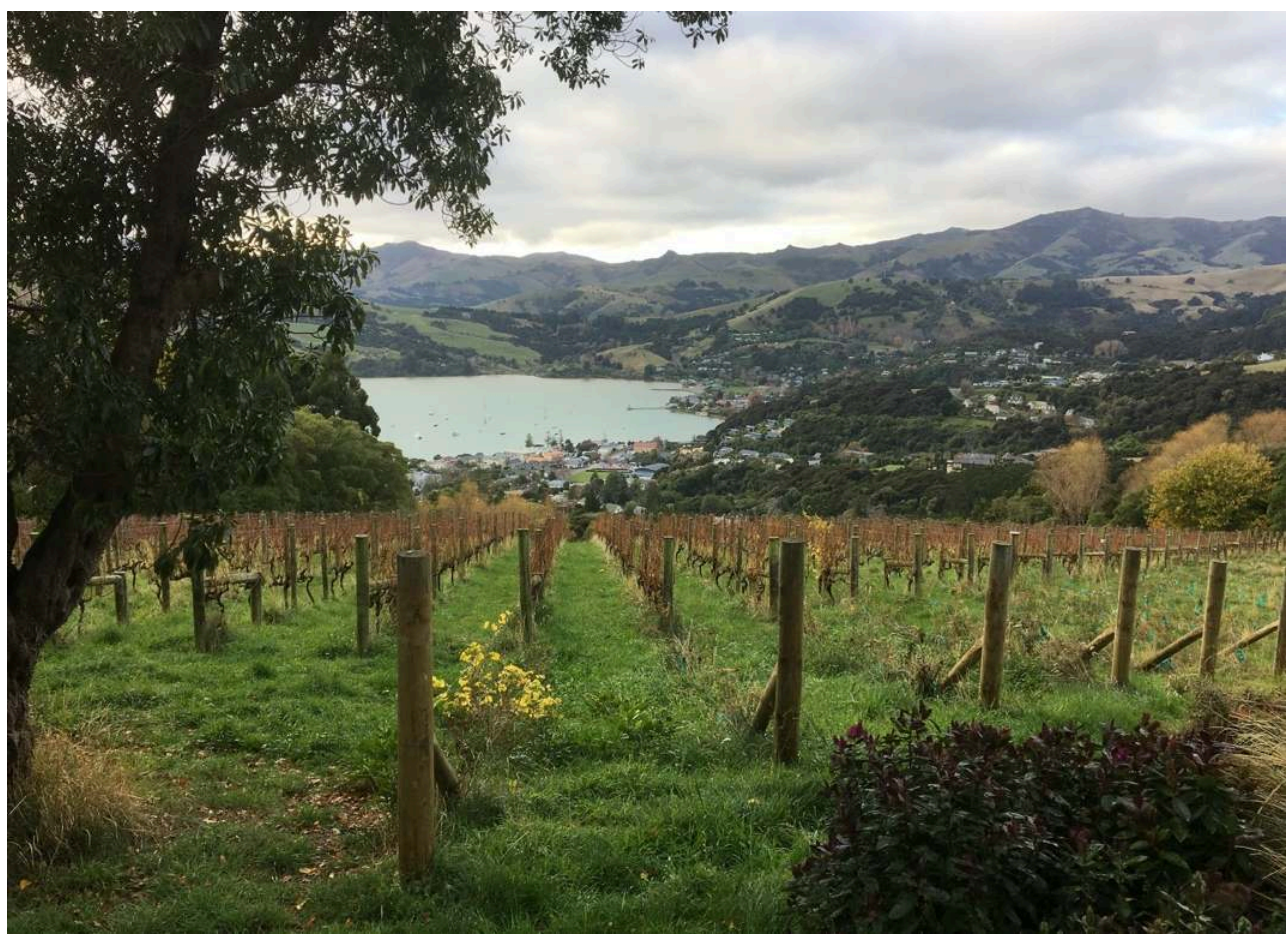

Parcelle de vignes de pinot noir, pinot gris et riesling sur une petite exploitation familiale. Aucune irrigation n'est utilisée ici, fait rare en Nouvelle-Zélande. Le sous-sol est d'origine volcanique, la presqu'île de Banks étant formée de deux anciens volcans.

Cliché : R. Hill, juin 2020.

Dans cet article, nous avons dressé le tableau d'une culture nationale qui s'est tournée vers le vin, et d'une industrie qui roule à deux vitesses. Même si le prix de revient du «bulk wine » ne représente que la moitié du prix de vin en bouteilles, cette première catégorie représente actuellement la moitié du vin exporté de la Nouvelle-Zélande (New Zealand Winegrowers, 2021). Les grandes entreprises sont fort pragmatiques dans leur intégration à cette filière internationale, et elles n'ont pas tort quand elles défendent les qualités de leur Sauvignon Blanc de Marlborough (évidemment bien fait et largement apprécié). Or, en demandant s'ils ont leur place à la table des pays producteurs de grands vins, comme l'a fait récemment le documentaire A Seat at the Table, les vignerons de Nouvelle-Zélande portent de plus en plus attention à leurs propres histoires, au génie de leurs lieux, et à ce qu'apporte leur culture à celle du vin. N'est-ce pas le même esprit qui anima les vignerons français du Moyen Âge, héritiers des traditions monastiques, ou celui des vignerons romains, héritiers des traditions grecques?

On ne peut nier la modernité de l'industrie viticole en Nouvelle-Zélande: elle est amplifiée par le manque d'anciennes familles viticoles et de vieux bâtiments (Schirmer, 2006). Mais l'intérêt pour le vin est tel que les acteurs de l'industrie s'engagent dans les tendances mondiales : ils élaborent des vins naturels, des vins de terroir, des vins de cépages rares, ainsi que ce sauvignon blanc qui se vend trop bien. Si dans certains territoires la tradition demeure une base de connaissances permettant l'innovation, la situation est inverse pour de nombreux vignerons en Nouvelle-Zélande. Beaucoup d'entre eux ont appris leur métier par un cours de formation, axé sur la science et la technologie. Nombre passent aussi par des stages en grandes entreprises où la 
technologie puissante est de mise. Or, forts de cette maitrise technique de la vinification, ils ont acquis la confiance d'expérimenter, d'apprendre, de laisser s'exprimer le terroir, et d'élaborer de nouvelles traditions. Ces tendances portent leurs fruits dans de nombreux domaines néo-zélandais, et ces fruits restent à découvrir.

\section{BIBLIOGRAPHIE}

Anderson K, Nelgen S et Pinilla V., 2017, Global wine markets, 1860-2016: a statistical compendium, Adelaïde, University of Adelaide Press.

Bailly A S., 2000, «Le temps des cépages : du terroir au système-monde », Annales de Géographie, $\mathrm{n}^{\circ}$ 614, p. 516-24.

Banks G., 2014, « What's in a name? Labels and branding in the New Zealand wine industry » dans Howland P J., Social, cultural and economic impacts of wine in New Zealand, Abingdon, Routledge, p. 120-36.

Beaven D W., 1977, Wines for dining, Christchurch (NZ), Whitcoulls.

Boltanski L et Esquerre A., 2017, Enrichissement : une critique de la marchandise, Paris, Gallimard.

Cappeliez S., 2017, « How well does terroir travel? Illuminating cultural translation using a comparative wine case study ", Poetics, $\mathrm{n}^{\circ}$ 65, p. 24-36.

Ciatti., 2021, Global Market Report, vol. 12, n 4, Rapport, Novato (CA).

Cooper M., 2021, « Tipping point », New Zealand Listener, 27 février 2021, p. 18-25.

Crenn C et Téchoueyres I., 2005, « Territoire viticole, patrimoine et distinction : le cas du Pays

foyen », Sud-Ouest Européen, $\mathrm{n}^{\circ} 19$, p. 49-55.

Dalley B., 2008, « Wine » dans Te Ara - the Encyclopedia of New Zealand (URL: https://teara.govt.nz/ en/wine).

Deloitte., 2019, Growing Smarter: Wine industry benchmarking and insights 2018, New Zealand, Rapport, Auckland.

Demossier M., 2012, « The Europeanization of Terroir: consuming place, tradition and Authenticity » dans Friedman R et Thiel M., European identity and culture: narratives of transnational belonging, Farnham, Ashgate.

Dion R., 1959, Histoire de la vigne et du vin en France des origines au XIXe siècle, Paris, Chez l'auteur.

Fassier-Boulanger S., 2014, « Les mutations de la filière viticole en France », Revue Géographique de l'Est, vol. 54, nº 1-2 (DOI : https://doi.org/10.4000/rge.5165).

Gade D W., 2004, « Tradition, territory, and terroir in French viniculture: Cassis, France, and appellation contrôlée ", Annals of the Association of American Geographers, vol. 94, n 4, p. 848-67. Hawke's Bay Today., 2008, « Beware! The Sauvalanche season is here! », Hawke's Bay Today, 9 août 2008, p.7. 
Héritier S., 2013 « Le patrimoine comme chronogenèse : réflexions sur l'espace et le temps » Annales de Géographie, $\mathrm{n}^{\circ}$ 689, p. 3-23.

Hill R., 2016, « Wine's united kingdom », Food 2.0 Lab (Site web) (URL: https://food20.fr/winesunited-kingdom/?lang=en).

Hill R., 2020, « Aptitude or adaptation: what lies at the root of terroir? », Geographical Journal, n ${ }^{\circ}$ 186, p. 346-50.

IPONZ [Intellectual Property Office of New Zealand]., 2021a., « North Canterbury 1016 », (Site web) (URL : https://www.iponz.govt.nz/about-ip/geographical-indications/register/northcanterbury/).

IPONZ [Intellectual Property Office of New Zealand]., 2021b, « The Geographical Indications Act », (Site web) (URL: https://www.iponz.govt.nz/about-ip/geographical-indications/the-gi-act/).

Laferté G., 2011, « The folklorization of French farming: marketing luxury wine in the interwar years », French Historical Studies, n 34, p. 680-712.

Legouy F et Boulanger S., 2015, Atlas de la vigne et du vin, Paris, Armand Colin.

Lewis N et Le Heron E., 2018, « New Zealand wine: seeking success beyond growth » dans Pawson E., The new biological economy: how New Zealanders are creating value from the land, Auckland, Auckland University Press, p. 116-36.

Moran W., 2000, «Culture et nature dans la géographie de l'industrie vinicole néo-zélandaise », Annales de Géographie, no 614-5, p. 525-51.

Moran W., 2016, New Zealand wine: the land, the vines, the people Auckland, Auckland University Press.

Murray W E et Overton J., 2011, Defining regions: the making of places in the New Zealand wine industry, Australian Geographer, vol. 42, n 4, p. 419-33.

New Zealand Winegrowers., 2020a, Annual Report 2020, Rapport, Auckland.

New Zealand Winegrowers., 2020b, New Zealand wine: a comprehensive guide to the regions and varieties, Rapport, Auckland.

New Zealand Winegrowers., 2020c, Vineyard register report, 2019-2022, Rapport, Auckland.

New Zealand Winegrowers., 2021, A Snapshot 2021: New Zealand Wine, Dépliant (URL: https:// www.nzwine.com/media/19215/2021-vr-snapshots-nz.pdf).

Overton J., 2020, « Landscapes of failure : why do some wine regions not succeed ? ", dans Myles C C., Fermented landscapes, Lincoln (NE), University of Nebraska Press, p. 57-82.

Pine B J et Gilmore J H, 1998, « Welcome to the experience economy », Harvard Business Review, juillet-août 1998, p. 97-105.

Pitte J-R., 2001, « La géographie du goût : entre mondialisation et enracinement local », Annales de Géographie, $\mathrm{n}^{\circ}$ 621, p. 487-508.

Rainer G, Puetz R, et Steiner C., 2019, " The emergence of new wine design practices: flexitanks and the assembling of bulk wine across global rural regions ", Geographical Journal (DOI: https:// doi.org/10.1111/geoj.12337).

Renall D., 2017, 100 Great New Zealand Wineries, Auckland, Bateman Publishing. 
Roudié P., 2007, « Le Terroir, mythe(s) or réalité(s)? », dans Actes du Colloque International sur les Terroirs, 9-12 mai 2007, Aix-en-Provence, p. 16-18.

Schirmer R., 2006, « Les paysages des vignobles d'Australie : de l'Arcadie au Jacob's Creek », SudOuest Européen, $\mathrm{n}^{\circ} 21$, p. 105-16.

Schirmer R., 2007, « Les vins du Nouveau Monde sont-ils a-géographiques ? », Bulletin de l'Association de Géographes Français, vol. 84, $\mathrm{n}^{\circ}$ 1, p. 65-80.

Schuster D, Jackson D et Tipples R., 2002, Canterbury grapes and wines, 1840-2002, Christchurch (NZ), Shoal Bay Press.

Tongue In Groove., 2021, « Pinot noir Cabal vineyard 2015 », (Site web), (URL: https:// tongueingroove.co.nz/shop/2014-cabal-pinot-noir/).

Trubek A., 2008, The taste of place, Berkeley (CA), University of California Press.

Van Waerbeke J., 2001, « La patrimonialisation du paysage », Travaux de l'Institut de Géographie de Reims, $\mathrm{n}^{\circ}$ 105-106, p. 31-36.

Vinography., 2017, « Turangawaewae: A Maori Expression of Terroir », Vinography (Site web), (URL: http://www.vinography.com/archives/2017/02/turangawhaewhae_a_maori_expres.html).

Whistling Buoy., 2021, « Whistling Buoy vineyard », (Site web) (URL: https:// www.whistlingbuoy.co.nz/index.php/en/about/vineyard).

Wynyard M., 2019, « 'Not One More Bloody Acre': Land Restitution and the Treaty of Waitangi Settlement Process in Aotearoa New Zealand ", Land, $\mathrm{n}^{\circ}$ 8, p. 162-176.

\section{NOTES}

1. Traduction de Rory Hill.

2. Ibid.

3. Les GI sont souvent considérées comme l'équivalent des AOP. Or, en réalité elles ressemblent plus aux IGP, puisqu'elles n'ont pas de cahiers de charges stricts, et les aires des appellations sont souvent vastes, jusqu'à englober une région entière.

4. Traduit par Rory Hill.

5. Ibid.

6. Ibid.

\section{RÉSUMÉS}

Les premières vignes furent plantées en Nouvelle-Zélande par les Européens qui sont arrivés au cours du XIX ${ }^{\mathrm{e}}$ siècle. Aujourd'hui, le pays soutient une véritable industrie viti-vinicole, et profite d'une grande notoriété pour son vin blanc issu du cépage Sauvignon blanc. Or, le succès de ce vin blanc de la région de Marlborough cache les sorts variés de nombreux petits producteurs ailleurs dans le pays. Cet article explique la situation actuelle, où $93 \%$ du vin néo-zélandais est produit par seulement $7 \%$ des domaines du pays. L'identification et la mise en culture des terroirs 
viticoles s'est effectuée sous des politiques agricoles néolibérales, et en accompagnant le développement graduel d'une culture populaire du vin. Chez les vignerons, l'excellence technique et l'adoption rapide des innovations n'empêchent pas de s'intéresser aux nouveaux terroirs et à l'adaptation des cépages divers aux conditions climatiques variables du pays. L'instauration récente d'un système de « Geographical Indications » (GI ; variant du système des $\mathrm{AOP}$ ) aide à la mise en avant des petites régions viticoles, bien que le mot terroir ne se diffuse pas partout dans la culture informelle et anglophone du pays. Des éléments de la culture Maori indigène commencent à se présenter dans le monde du vin néo-zélandais, et certains vins acquièrent des réputations qui seront, peut-être, les références de l'avenir.

Whilst the first grapes were only planted in New Zealand with the arrival of Europeans in the 19th century, the country's wine industry has rapidly grown. Today, it is globally recognized for its Sauvignon Blanc, which exploded in popularity in the 1980s. The success of Marlborough Sauvignon Blanc, however, and of several of the large estates which produce it, masks the variable fortunes of hundreds of smaller wine producers. In this article, primary and secondary research is used to reveal the story behind the current situation where $93 \%$ of New Zealand's wine is made by just $7 \%$ of its producers. The historical identification of ideal grape-growing territories is presented alongside the influence of neoliberal agricultural and trade policies, and the gradual development of wine culture in the young country. For producers, technical excellence and early adoption of innovations are accompanied by strong interest in finding propitious terroirs and adapting grape varieties to the varied terrain, meso and micro-climates of the country. The recent instauration of a system of Geographical Indications (GIs) is helping to demarcate the country's wine regions, and providing a shared identity for small producers, though the term "terroir" is not entirely domesticated within New Zealand's relatively informal culture. Some producers are looking for ways to include traditional Maori concepts in the development of a sense of place and a sense of purpose for the wine industry, and several distinctive wines are building reputations in the hope of becoming the classics of the future.

\section{INDEX}

Mots-clés : Nouvelle-Zélande, vin, terroir, Sauvignon blanc, North Canterbury, Marlborough, Maori, tūrangawaewae

Keywords : New Zealand, wine, terroir, Sauvignon Blanc, North Canterbury, Marlborough, Maori, tūrangawaewae

\section{AUTEUR}

\section{RORY HILL}

Eastern Institute of Technology, Napier, Nouvelle-Zélande

rhill@eit.ac.nz 